\title{
Vaccenic acid favourably alters immune function in obese JCR:LA-cp rats
}

\author{
Heather J. Blewett, Christopher A. Gerdung, Megan R. Ruth, Spencer D. Proctor and Catherine J. Field* \\ Alberta Institute for Human Nutrition, Department of Agricultural, Food and Nutritional Sciences, University of Alberta, \\ Edmonton, AB, T6G 2E1, Canada
}

(Received 4 September 2008 - Revised 4 December 2008 - Accepted 17 December 2008 - First published online 16 February 2009)

Vaccenic acid (VA) is a ruminant-derived trans-fat and precursor of conjugated linoleic acid (CLA). The objective of the present study was to explore the effects of VA on immune function in a model of the metabolic syndrome, JCR:LA- $c p$ rats. Lean $(2: 1$ mix of $+/ c p$ and $+/+)$ and obese $(\mathrm{cp} / \mathrm{cp})$ rats, aged 8 weeks, were fed a control $(0 \% \mathrm{VA})$ or a VA diet $(1.5 \%$ (w/w) VA) for 3 weeks (twenty rats per group). Splenocytes and mesenteric lymph node (MLN) immune cell phenotypes (flow cytometry), ex vivo cytokine production (ELISA) and phospholipid fatty acid concentrations were measured. Obese rats had higher proportions of splenic macrophages, total T-cells, helper T-cells (total and percentage $\mathrm{CD} 25^{+}$), cytotoxic T-cells (total and percentage $\mathrm{CD} 25^{+}$) and produced higher concentrations of IL-6 to concanavalin A (ConA) compared with lean rats. Obese rats had lower proportions of MLN T-cells, new T-cells $\left(\mathrm{CD}^{+} \mathrm{CD} 90^{+}\right)$and cytotoxic T-cells, but higher proportions of helper cells that were $\mathrm{CD} 45 \mathrm{RC}^{+}, \mathrm{CD} 25^{+}$and $\mathrm{CD} 41 \mathrm{lo}$, and produced higher concentrations of IL-2, IL-10, interferon $\gamma$ and TNF $\alpha$ in response to ConA compared with lean rats. VA was higher in plasma phospholipids and both VA and CLA (cis-9, trans-11) were higher in MLN phospholipids compared with control-fed rats. Lean VA-fed rats had lower proportions of MLN and splenocyte CD45RC ${ }^{+}$helper cells, and helper T-cells. Splenocytes from VA-fed rats produced 16-23\% less IL-2, IL-10 and TNF $\alpha$ compared with controls. VA normalised production of MLN IL-2 and TNF $\alpha$ in obese rats to levels similar to those seen in lean rats. These results indicate that dietary VA favourably alters the pro-inflammatory tendency of mesenteric lymphocytes from JCR:LA-cp rats.

Vaccenic acid: Obesity: Immune: JCR:LA-cp rats

Trans-fat has been linked with an increased risk of heart disease and various health organisations have recommended dietary intake be reduced ${ }^{(1,2)}$. However, trans-fatty acids (fats containing at least one double bond in the trans configuration) are a heterogeneous group of molecules, probably having differing effects on health. Trans-fats consumed in the diet are produced either during the partial hydrogenation of vegetable oils or are present naturally in ruminant products (beef and dairy) by the biohydrogenation of PUFA by microorganisms in the rumen ${ }^{(3,4)}$. Emerging evidence supports a distinction between industrially produced trans-fats (i.e. elaidic acid) and trans-fats produced naturally in beef and milk (i.e. conjugated linoleic acid (CLA) and vaccenic acid (VA)) based on their differing effects on health ${ }^{(5)}$. A number of different health effects have been ascribed to the 'natural' trans-fatty acid CLA (for a review, see Bhattacharya et al. ${ }^{(6)}$ ) and producers have been successful at increasing the CLA content of milk and beef through various feeding strategies (for reviews, see Lock \& Bauman ${ }^{(4)}$ and Mir et al. $\left.{ }^{(7)}\right)$. However, as the CLA content of beef and dairy increases, so does the VA (trans-11-18:1) content of the product $^{(8,9)}$. Interest in VA has increased based on its ability to be converted to the cis-9, trans-11 form of CLA, including in humans ${ }^{(10)}$. It remains to be seen whether dietary VA shares the health benefits ascribed to CLA or the reputed deleterious health effects of the industrially produced trans-fatty acids.

Our group has recently reported hypotriacylglycerolaemic effects of dietary VA at $1.5 \%(\mathrm{w} / \mathrm{w})$ in the obese JCR:LA$c p$ rat $^{(11)}$. We also reported lower serum IL-10 concentrations with dietary VA in obese rats, suggesting an alteration in T-cell function ${ }^{(11)}$. Obesity has been associated with both impaired immune responses and an exaggerated pro-inflammatory response ${ }^{(12,13)}$. Researchers have shown that in healthy subjects dietary VA at $3 \mathrm{~g} / \mathrm{d}$ was incorporated into the cellular membranes of immune cells, but did not alter the phenotype of immune cells in the blood, phagocytic activity of granulocytes, or serum concentrations of cytokines, adipokines and eicosanoids $^{(14)}$. Ruth et al. ${ }^{(12)}$ have shown incorporation into membrane phospholipids and alterations in $\mathrm{T}$-cell function after feeding CLA (cis-9, trans-11) in an obese rat model. The mechanistic effects of dietary VA on the immune abnormalities associated with obesity and insulin resistance are not known.

To explore the potential immunomodulatory properties of $\mathrm{VA}$ in a chronic inflammatory state we examined the effects of both obesity and dietary VA on splenic and mesenteric lymph node (MLN) immune cell phenotypes and function, using an established rat model of the metabolic syndrome,

Abbreviations: CLA, conjugated linoleic acid; ConA, concanavalin A; IFN, interferon; LPS, lipopolysaccharide; MLN, mesenteric lymph node; VA, vaccenic acid. * Corresponding author: Dr Catherine J. Field, fax +1 780492 2011, email Catherine.Field@ualberta.ca 
the JCR:LA-cp rat. The fatty acid composition of the two diets was designed in such a way that the major difference was in the presence or absence of VA. We were interested in whether there would be incorporation of VA and cis-9, trans-11-CLA (due to conversion from VA) into immune cell phospholipids and whether this would influence the concentration of proliferative (IL-2), inflammatory (IL-1 $\beta$, IL-6, $\mathrm{TNF} \alpha$, interferon (IFN) $\gamma$ ) and regulatory (IL-10) cytokines produced from the VA-fed rats compared with controls. The JCR:LA-cp rat spontaneously develops obesity, insulin resistance, dyslipidaemia and atherosclerosis if homozygous for the autosomal recessive corpulent $(c p)$ gene $(c p / c p)^{(15)}$. Rats that are heterozygous $(+/ c p)$ or homozygous normal $(+/+)$ are lean and metabolically normal ${ }^{(15)}$. Therefore, this animal model allows us to examine the effects of dietary $\mathrm{VA}$ in both a healthy animal and one with severe insulin resistance and at risk of atherosclerosis. We have recently reported that the JCR:LA-cp rat exhibits serum markers of chronic low-grade inflammation ${ }^{(11)}$ similar to that reported in humans with the metabolic syndrome ${ }^{(16)}$; however, little is known about the impact of obesity on T-cell function in this animal model.

\section{Experimental methods}

Animals and diets

Male JCR:LA- $c p$ rats, aged 6 weeks, were obtained from our established breeding colony maintained at the University of Alberta. Rats were housed individually and animal care was provided in accordance with a protocol approved by the Animal Policy and Welfare Committee of the Faculty of Agriculture, Forestry and Home Economics at the University of Alberta. For 2 weeks, rats were fed standard rat chow (Lab diet 5001; PMI Nutrition International, Brentwood, MO, USA) with $1 \%(\mathrm{w} / \mathrm{w})$ cholesterol (Sigma, Oakville, $\mathrm{ON}$, Canada) to exacerbate inflammatory processes and accelerate atherosclerosis ${ }^{(11)}$. At age 8 weeks, obese $(c p / c p ; n 20)$ and lean rats $(2: 1$ mix of heterozygous $(+/ c p)$ and homozygous normal $(+/+) ; n 20)$ were randomly assigned to one of two nutritionally adequate basal diets: a control diet $(0 \%$ $\mathrm{VA})$ or a diet supplemented with VA $(1.5 \%$, w/w) for 3 weeks. Both diets were prepared at the University of Alberta using a nutritionally adequate basal diet (Harlan Teklad, Madison, WI, USA) with a $15 \%(\mathrm{w} / \mathrm{w})$ fat mixture as described previously ${ }^{(11)}$. Both diets contained (per kg): $267 \mathrm{~g}$ casein, $539 \mathrm{~g}$ carbohydrate ( $222 \mathrm{~g}$ starch, $231 \mathrm{~g}$ dextrose) and $150 \mathrm{~g}$ fat. Both diets provided a PUFA:SFA ratio of $0 \cdot 6$. The diets were carefully designed to have similar fatty acid compositions, differing only in VA (trans-11-18:1) and oleic acid (cis-9-18:1). Dietary fatty acid composition measured by GC is shown in Table 1 . Both feed intake and body weight were recorded weekly over the 3 -week feeding period.

\section{Tissue collection}

Rats were anaesthetised with isoflurane and exsanguinated via cardiac puncture. The spleen and MLN were removed aseptically, placed in PBS and processed immediately.
Table 1. Fatty acid composition of diets $(\mathrm{g} / 100 \mathrm{~g}$ total fatty acids)*

\begin{tabular}{lcc}
\hline Fatty acid & Vaccenic acid diet & Control diet \\
\hline $14: 0$ & $0 \cdot 1$ & $0 \cdot 1$ \\
$16: 0$ & $10 \cdot 7$ & $12 \cdot 9$ \\
$18: 0$ & $37 \cdot 0$ & $35 \cdot 5$ \\
$18: 1$ (cis-9) & $11 \cdot 8$ & $20 \cdot 9$ \\
Vaccenic acid (trans-11) & $9 \cdot 1$ & $0 \cdot 4$ \\
$18: 2 n-6$ & $28 \cdot 2$ & $26 \cdot 0$ \\
$18: 3 n-3$ & $2 \cdot 0$ & $2 \cdot 6$ \\
$20: 0$ & $0 \cdot 4$ & $0 \cdot 3$ \\
$20: 1$ & $\mathrm{ND}$ & $0 \cdot 7$ \\
$20: 5 n-3$ & $\mathrm{ND}$ & $\mathrm{ND}$ \\
$22: 0$ & $0 \cdot 3$ & $0 \cdot 2$ \\
$22: 2 n-6$ & $0 \cdot 1$ & $\mathrm{ND}$ \\
$22: 5 n-6$ & $0 \cdot 2$ & $\mathrm{ND}$ \\
$\sum$ SFA & $48 \cdot 5$ & $49 \cdot 3$ \\
$\sum$ MUFA & $20 \cdot 9$ & $22 \cdot 1$ \\
$\sum$ PUFA & $30 \cdot 5$ & $28 \cdot 6$ \\
$\sum n-6$ PUFA & $28 \cdot 5$ & $26 \cdot 1$ \\
$\sum n-3$ PUFA & $2 \cdot 0$ & $2 \cdot 6$ \\
PUFA:SFA ratio & $0 \cdot 6$ & $0 \cdot 6$ \\
$n-6: n-3$ ratio & $14 \cdot 3$ & $10 \cdot 0$ \\
\hline
\end{tabular}

ND, not detectable.

* Diets contained $150 \mathrm{~g}$ fat $/ \mathrm{kg}$ from a mixture of sources (sunflower-seed oil, flaxseed oil, soya, tallow, olive oil (control diet) and vaccenic acid (vaccenic acid diet)). Fatty acids not reported: $12: 0 ; 16: 1 ; 17: 0 ; 17: 1$; $20: 2 ; 24: 0$.

\section{Isolation of immune cells and estimate of proliferation}

Immune cells were isolated from the spleen and MLN as previously described ${ }^{(17)}$. Briefly, single cell suspensions were obtained by disrupting tissue through a nylon mesh screen in Krebs-Ringer HEPES buffer with bovine serum albumin (5 g/l) (Sigma, Oakville, ON, Canada). Erythrocytes were lysed with ammonium chloride lysis buffer (155 mM$\mathrm{NH}_{4} \mathrm{Cl}, \quad 0.1 \mathrm{~mm}-\mathrm{EDTA}, 10 \mathrm{~mm}-\mathrm{KHCO}_{3}$; Fisher Scientific, Edmonton, AB, Canada). Cells were subsequently washed and re-suspended in complete culture media (Roswell Park Memorial Institute (RPMI) 1640 media supplemented with $5 \%(\mathrm{v} / \mathrm{v})$ heat-inactivated fetal calf serum, $25 \mathrm{mM}$-HEPES, $2.5 \mathrm{mM}$-2-mercaptoethanol and $1 \%$ antibiotic/antimycotic ( $\mathrm{pH} 7 \cdot 4)$; Invitrogen, Burlington, ON, Canada). Cell concentration and viability were determined using a haemocytometer and trypan blue dye exclusion, respectively.

\section{Plasma and mesenteric lymph node phospholipid fatty acid composition}

Lipids were extracted from plasma and MLN cells by a modified Folch method as previously described ${ }^{(18)}$. C15:0 TAG standard $(100 \mu \mathrm{g} / \mathrm{ml})$ was added to plasma samples as an internal control. Total phospholipids were separated on silica $\mathrm{G}$ plates and visualised with 8-anilino-1-naphthalenesulfonic acid under UV light using the appropriate standards ${ }^{(19)}$. The phospholipid silica band was scraped and used to prepare fatty acid methyl esters. The base-catalysed method using sodium methoxide was used to prepare methyl esters as described elsewhere ${ }^{(20)}$. Prepared phospholipid fatty acid methyl esters were flushed with $\mathrm{N}_{2}$ and stored at $-35^{\circ} \mathrm{C}$ until analysis by GC. Fatty acids were then analysed by automated GLC (Varian 3800; Varian Inc., Mississauga, ON, 
Canada) using a $100 \mathrm{~m}$ CP-Sil 88 fused capillary column (Varian Inc.) as described elsewhere ${ }^{(20)}$.

\section{Phenotyping}

Monoclonal antibodies against rat CD3 (PE label, clone G4.18, isotype $\mathrm{IgG}_{3, \mathrm{k}}$ ), CD4 (APC label, clone OX-35, isotype mouse $\mathrm{IgG}_{2, \mathrm{k}}$ ), CD11b/c (PE label, clone OX-42, isotype $\operatorname{IgG}_{2 \mathrm{a}, \mathrm{k}}$ ), CD25 (PE label, clone OX-39, isotype mouse $\operatorname{IgG}_{1, \mathrm{k}}$ ), CD28 (FITC label, clone JJ319, isotype mouse $\operatorname{IgG}_{1, \mathrm{k}}$ ), CD45RC (FITC label, clone OX-22, isotype mouse $\mathrm{IgG}_{1, \mathrm{k}}$ ) and CD90 (PerCP label, clone OX-7, isotype mouse $\mathrm{IgG}_{1, \mathrm{k}}$ ) were obtained from BD BioScience (Mississauga, ON, Canada). CD8 (RPE-Cy5 label, clone OX-8, isotype mouse $\operatorname{IgG}_{1, \mathrm{k}}$ ) and CD152 (FITC label, clone WKH203, isotype mouse $\mathrm{IgG}_{1}$ ) were obtained from AbD Serotec (Raleigh, NC, USA). Immune cell phenotypes were determined by flow cytometry using isolated immune cells as previously described ${ }^{(19)}$. Fluorescent antibodies were used in the following combinations: $\quad \mathrm{CD} 28 / \mathrm{CD} 3 / \mathrm{CD} 8 / \mathrm{CD} 4 ; \quad \mathrm{CD} 152 / \mathrm{CD} 25 / \mathrm{CD} 8 / \mathrm{CD} 4$; CD45RC/CD3/CD90/CD4; CD45RC/CD8/CD4; CD11b/c; OX-12. Splenic and MLN cells were then washed twice with PBS supplemented with fetal calf serum $(4 \mathrm{~g} / \mathrm{l})$ and fixed with $1 \%(\mathrm{w} / \mathrm{v})$ paraformaldehyde. Data were acquired on a FACSCalibur four-colour flow cytometer (Becton Dickinson, San Jose, CA, USA) and analysed using CellQuest software (Becton Dickinson, San Jose, CA, USA). Unstained cells were used to assess autofluorescence, while single colour samples were employed to adjust colour compensation.

\section{Cytokine determination}

Splenocytes $\left(1.25 \times 10^{6}\right.$ cells $\left./ \mathrm{ml}\right)$ were incubated for $48 \mathrm{~h}$ with $5 \% \mathrm{CO}_{2}$ in either the presence or absence of concanavalin A (ConA; $2.5 \mu \mathrm{g} / \mathrm{ml}$; MP Biomedicals, Montreal, Quebec, Canada) or lipopolysaccharide (LPS; $100 \mu \mathrm{g} / \mathrm{ml}$, Sigma). Cells from the MLN were incubated in either the presence or absence of ConA. After the $48 \mathrm{~h}$ incubation, cells were centrifuged for $10 \mathrm{~min}$ at $1000 \mathrm{rpm}$. Supernatant fractions were collected and stored at $-80^{\circ} \mathrm{C}$ until analysis. The concentration (detection limits in parentheses) of IL-1 $\quad(62 \cdot 5-4000 \mathrm{pg} / \mathrm{ml}), \quad$ IL-2 $\quad(23 \cdot 4-1500 \mathrm{pg} / \mathrm{ml})$, IL-6 $\quad(78-5000 \mathrm{pg} / \mathrm{ml}), \quad$ IL-10 $\quad(15 \cdot 6-1000 \mathrm{pg} / \mathrm{ml}), \quad$ IFN $\gamma$ $(31.25-2000 \mathrm{pg} / \mathrm{ml})$ and $\mathrm{TNF} \alpha(31.2-2000 \mathrm{pg} / \mathrm{ml})$ in the supernatant fractions were determined using a commercially available ELISA kit (IL-1 $\beta$, IL-2, Medicorp Inc., Montreal, Quebec; IL-6, IL-10, IFN $\gamma, \mathrm{TNF} \alpha$, BD Biosciences, Mississauga, ON, Canada). All samples were analysed in duplicate with $\mathrm{CV}<10 \%$. All plates were read according to the manufacturer's specifications in a microplate reader (SpectraMax 190; Molecular Device, Sunnyvale, CA, USA).

\section{Statistical analysis}

All statistical analyses were conducted using SAS statistical software (version 9.1; SAS Institute Inc., Cary, NC, USA). Data are expressed as least squares means with their standard errors. Normality of the distribution was tested and data were log-transformed if necessary. Data were analysed by two-way ANOVA for main effects of diet and genotype as well as the interaction between diet and genotype. Differences among groups were identified using LSMeans. For all results, $P<0.05$ was considered statistically significant.

\section{Results \\ Effect of obesity and vaccenic acid on feed intake, body weight and organ weights}

Obese $(c p / c p)$ rats had significantly higher final body weight (447 (SEM 5) v. 303 (SEM 7) g; $P<0.0001$ ) and average feed intake (38 (SEM 0.6) v. 20 (SEM 0.5) g/d; $P<0.0001)$ compared with lean rats. There was no effect of VA on body weight or feed intake in either obese or lean rats during the experimental treatment period. Absolute spleen weight did not differ between diet groups or genotype (0.77 (SEM 0.02) g; n 40), but when corrected for body weight, obese rats had lower spleen weight compared with lean rats (1.8 (SEM 0.04) v. 2.5 (SEM 0.06) $\mathrm{mg} / \mathrm{g}$ body weight; $P<0.001$ ), respectively. There was no net effect of obesity on the number of cells per spleen. However, obese rats had a lower number of cells per $g$ of spleen compared with lean rats $\left(2.0 \times 10^{8}\left(\right.\right.$ SEM $\left.0.1 \times 10^{8}\right)$ v. $2.5 \times 10^{8}\left(\right.$ SEM $\left.\left.0.2 \times 10^{8}\right) ; P=0.02\right)$, respectively. We also observed a significant interaction between diet and genotype $(P=0.01)$. Lean rats fed VA had higher spleen weights per $\mathrm{g}$ of body weight compared with lean rats fed the control diet $(2.7$ (SEM 0.08) v. 2.4 (SEM 0.07) $\mathrm{mg} / \mathrm{g} ; \quad P=0.007$ ), while there was no significant difference between the obese rats fed the VA and control diets. Despite no effect of diet on absolute spleen weight, rats fed VA had a lower number of splenocytes per spleen compared with controls $\left(1.5 \times 10^{8}\right.$ $\left(\right.$ SEM $\left.0.1 \times 10^{8}\right)$ cells $v .1 .9 \times 10^{8}\left(\mathrm{SEM} 0.1 \times 10^{8}\right)$ cells; $P=0 \cdot 01$ ), respectively.

\section{Plasma and mesenteric lymph node phospholipid fatty acid composition}

Effect of obesity. Obese rats had higher plasma phospholipid concentrations of all fatty acids compared with lean rats (except 24:1n-9) (Table 2). There was no difference in the plasma phospholipid PUFA:SFA ratio between obese and lean rats. Interestingly, obese rats had a lower $n-6: n-3$ PUFA ratio compared with lean rats. Despite equivalent dietary composition, obese rats had a higher proportion of MLN phospholipid fatty acids as 16:0, 16:1 (cis-9), 18:1 (cis-11), 20:4n-6, 20:1n-9, $22: 5 n-3$, $22: 6 n-3$, and a lower proportion as $18: 1$ (cis-9) and $18: 2 n-6$ compared with lean rats (Table 3 ).

Effect of diet. VA-fed rats had a $1237 \%$ and $1200 \%$ higher concentration of VA in plasma and MLN phospholipids compared with rats fed the control diet (Tables 2 and 3). Rats fed VA also had $0.25 \%$ of total fatty acids as CLA in MLN phospholipids, while CLA was not detectable in the MLN of control-fed rats. The VA-fed rats had higher concentrations of 18:0 in plasma phospholipids compared with rats fed the control diet.

Effect of vaccenic acid in lean $\mathrm{v}$. obese rats. Obese VA-fed rats had higher concentrations of 16:0 in MLN and plasma phospholipids compared with obese control-fed rats, but there was no difference between dietary treatments in the lean rats. Lean VA-fed rats had higher concentrations of 16:1 (cis-9), $17: 0$ and $18: 2 n-6$ in plasma phospholipids and lower concentrations of 18:1 (cis-9) in MLN phospholipids compared with 
Table 2. The effect of dietary vaccenic acid (VA) on plasma phospholipid fatty acid composition in JCR:LA/cp rats (Mean values with their standard errors of ten rats per group)

\begin{tabular}{|c|c|c|c|c|c|c|c|c|c|c|}
\hline \multirow[b]{3}{*}{ Fatty acid $(\mu \mathrm{g} / \mathrm{ml})$} & \multicolumn{4}{|c|}{ Lean animals } & \multicolumn{4}{|c|}{ Obese animals } & & \\
\hline & \multicolumn{2}{|c|}{ VA diet } & \multicolumn{2}{|c|}{ Control diet } & \multicolumn{2}{|c|}{ VA diet } & \multicolumn{2}{|c|}{ Control diet } & \multicolumn{2}{|c|}{ Significance* } \\
\hline & Mean & SEM & Mean & SEM & Mean & SEM & Mean & SEM & Diet & Genotype \\
\hline $14: 0$ & $1 \cdot 8$ & 0.7 & 0.9 & 0.4 & 2.5 & 0.3 & $1 \cdot 8$ & $0 \cdot 6$ & NS & 0.005 \\
\hline $16: 0$ & $87 \cdot 1^{\mathrm{c}}$ & 5.4 & $65 \cdot 7^{c}$ & $7 \cdot 3$ & $242^{\mathrm{a}}$ & $18 \cdot 3$ & $183^{\mathrm{b}}$ & $26 \cdot 9$ & 0.04 & $<0.0001$ \\
\hline $16: 1$ (cis-9) & $1.6^{\mathrm{b}}$ & 0.6 & $0.8^{\mathrm{c}}$ & 0.3 & $3.5^{\mathrm{a}}$ & 0.3 & $2 \cdot 9^{\mathrm{a}}$ & 0.4 & NS & $<0.0001$ \\
\hline $17: 0$ & $1 \cdot 3^{\mathrm{b}}$ & 0.1 & $0.9^{c}$ & 0.1 & $3 \cdot 2^{\mathrm{a}}$ & 0.5 & $2 \cdot 2^{\mathrm{a}}$ & 0.5 & NS & $<0.0001$ \\
\hline $18: 0$ & 252 & $42 \cdot 6$ & 157 & 23.4 & 899 & 256 & 598 & 138 & 0.03 & $<0.0001$ \\
\hline VA (trans-11) & 9.4 & $1 \cdot 6$ & 0.7 & 0.2 & $16 \cdot 0$ & $2 \cdot 9$ & $1 \cdot 2$ & 0.3 & $<0.0001$ & 0.05 \\
\hline $18: 1$ (cis-9) & $13 \cdot 1$ & $0 \cdot 6$ & $12 \cdot 0$ & $1 \cdot 0$ & $39 \cdot 0$ & 3.0 & 37.4 & $6 \cdot 0$ & NS & $<0.0001$ \\
\hline $18: 1$ (cis-11) & 3.0 & $0 \cdot 1$ & $2 \cdot 7$ & 0.2 & $6 \cdot 2$ & 0.8 & $7 \cdot 6$ & 1.0 & NS & $<0.0001$ \\
\hline $18: 2 n-6$ & $70 \cdot 8^{\mathrm{b}}$ & $4 \cdot 3$ & $49 \cdot 0^{c}$ & 4.0 & $131^{\mathrm{a}}$ & $18 \cdot 7$ & $109^{a}$ & $13 \cdot 1$ & 0.01 & $<0.0001$ \\
\hline CLA (cis-9, trans-11) & ND & - & ND & - & ND & - & ND & - & NS & NS \\
\hline $20: 3 n-6$ & 0.2 & 0.2 & 0.4 & 0.2 & $17 \cdot 7$ & 1.9 & $16 \cdot 0$ & 1.9 & NS & $<0.0001$ \\
\hline $20: 4 n-6$ & 79.5 & $5 \cdot 6$ & $62 \cdot 0$ & $4 \cdot 2$ & 157 & $19 \cdot 4$ & 155 & $11 \cdot 7$ & NS & $<0.0001$ \\
\hline $24: 1 n-9$ & 0.5 & $0 \cdot 1$ & 0.5 & 0.1 & 0.7 & 0.2 & 0.7 & 0.2 & NS & NS \\
\hline $22: 4 n-6$ & 0.5 & 0.3 & 0.3 & 0.1 & 1.4 & 0.3 & 1.3 & 0.3 & NS & $<0.0001$ \\
\hline $22: 5 n-3$ & 0.7 & 0.2 & 0.7 & 0.1 & 3.4 & 0.9 & $2 \cdot 3$ & 0.5 & 0.05 & $<0.0001$ \\
\hline $22: 6 n-3$ & $7 \cdot 1$ & 0.7 & $5 \cdot 7$ & 0.6 & $22 \cdot 9$ & $4 \cdot 8$ & $18 \cdot 7$ & $1 \cdot 8$ & NS & $<0.0001$ \\
\hline$\Sigma$ SFA & 342 & $46 \cdot 6$ & 224 & $30 \cdot 3$ & 1147 & 270 & 786 & 162 & 0.02 & $<0.0001$ \\
\hline$\Sigma$ MFA & $27 \cdot 6$ & $1 \cdot 7$ & $16 \cdot 7$ & 1.4 & 65.4 & $5 \cdot 3$ & $49 \cdot 7$ & $7 \cdot 1$ & $<0.0001$ & $<0.0001$ \\
\hline$\Sigma$ PUFA & 159 & 8.5 & 118 & $6 \cdot 7$ & 334 & $27 \cdot 3$ & 302 & $22 \cdot 7$ & NS & $<0.0001$ \\
\hline$\sum n-6$ & 151 & $8 \cdot 0$ & 112 & $6 \cdot 2$ & 307 & $24 \cdot 0$ & 281 & $21 \cdot 2$ & NS & $<0.0001$ \\
\hline$\sum n-3$ & 7.8 & $0 \cdot 8$ & 6.4 & 0.8 & $26 \cdot 3$ & $5 \cdot 7$ & $21 \cdot 0$ & $2 \cdot 1$ & NS & $<0.0001$ \\
\hline PUFA:SFA ratio & 0.5 & 0.4 & 0.6 & 0.1 & 0.4 & 0.1 & 0.5 & $0 \cdot 1$ & NS & NS \\
\hline$n-6: n-3$ ratio & $20 \cdot 9$ & $2 \cdot 2$ & $19 \cdot 5$ & $2 \cdot 7$ & $17 \cdot 2$ & 3.0 & $14 \cdot 0$ & $1 \cdot 1$ & NS & 0.02 \\
\hline
\end{tabular}

NS, $P>0.05$; ND, not detectable.

a,b,c Mean values within a row with unlike superscript letters were significantly different $(P<0.05$; LSMeans).

* Significant main effects (diet and genotype) were determined by two-way ANOVA. There were no significant interactions between diet and genotype.

lean control-fed rats, but there was no difference between dietary treatment groups in the obese rats.

Diet $\times$ genotype effect. There was no interaction between diet and genotype on plasma and MLN phospholipid fatty acid composition, except for the MLN phospholipid concentration of VA, $22: 6 n-3$ and $n-6: n-3$ ratio. VA was incorporated into MLN phospholipids to a lesser extent in the obese rats fed VA compared with lean rats fed VA. The lean rats fed VA had a higher MLN phospholipid n-6:n-3 ratio compared with lean rats fed the control diet, but there was no difference in the MLN phospholipid $n-6: n-3$ ratio between the obese rats fed the VA and control diets.

\section{Immune cell phenotypes}

Effect of obesity. The proportion of splenic T-cells was $6 \%$ higher in obese rats compared with lean rats due to a relative increase in both the proportion of helper $\left(\mathrm{CD}^{+} \mathrm{CD}^{+}\right)$and cytotoxic $\left(\mathrm{CD}^{+}{ }^{+} \mathrm{CD}^{+}\right)$T-cells (Table 4). Unlike the spleen, the proportion of MLN T-cells was 5\% lower in obese rats compared with lean rats due to an $18 \%$ decrease in the proportion of cytotoxic T-cells with no concomitant effect on the proportion of helper T-cells (Table 5). The ratio of helper: cytotoxic cells was $24 \%$ higher in obese compared with lean rats. Moreover, obese rats had a $21 \%$ higher proportion of splenic monocytes/macrophages $\left(\mathrm{CD} 11 \mathrm{~b} / \mathrm{c}^{+}\right)$compared with lean rats, but obesity did not affect the proportion of splenic B-cells $\left(\mathrm{OX}-12^{+}\right)$(Table 4). We also observed that obesity in this animal model did not affect the proportion of B-cells $\left(\mathrm{OX}-12^{+}\right)$, and macrophages $\left(\mathrm{CD} 11 \mathrm{~b} / \mathrm{c}^{+}\right)$in the MLN .

Effect of diet. There was no effect of VA on the proportion of T-cells, cytotoxic T-cells, B-cells or macrophages in the spleen and MLN of obese rats (Tables 4 and 5, respectively).

Effect of vaccenic acid in lean $\mathrm{v}$. obese rats. The lean rats fed VA had a $10 \%$ lower proportion of splenic and MLN helper T-cells compared with the lean rats fed the control diet, but there was no difference in the proportion of helper $\mathrm{T}$-cells between the dietary treatments in the obese rats.

Diet $\times$ genotype effect. There was no interaction between diet and genotype on immune cell phenotypes.

\section{Immune cell activation markers}

Effect of obesity. Obesity did not significantly affect the proportion of splenic helper or cytotoxic T-cells that expressed the co-stimulatory molecule $\mathrm{CD} 28\left(\mathrm{CD} 3^{+} \mathrm{CD} 4^{+} \mathrm{CD} 8^{-} \mathrm{CD} 28^{+}\right.$, 12.7 (SEM 0.4) \%, $n$ 40; $\mathrm{CD}^{+} \mathrm{CD}^{-} \mathrm{CD}^{+} \mathrm{CD} 28^{+}, 11.4$ (SEM 0.3$) \%, n 40$ ) or CD152 (CTLA-4; CD $4^{+} \mathrm{CD} 8^{-} \mathrm{CD} 152^{+}$, 0.36 (SEM 0.04) \%, $n 40 ; \mathrm{CD}^{-}{ }^{-} \mathrm{CD} 8^{+} \mathrm{CD} 152^{+}, 3.52$ (SEM 0.17) $\%, n 40)$. There was also no difference in the proportion of MLN helper and cytotoxic T-cells that expressed $\mathrm{CD} 28\left(\mathrm{CD}^{+-}\right.$ $\mathrm{CD}^{+}{ }^{+} \mathrm{CD} 8{ }^{-} \mathrm{CD} 28^{+}, 9.4$ (SEM 0.3) \%, $n$ 40; $\mathrm{CD}^{+}{ }^{+} \mathrm{CD} 4^{-} \mathrm{CD} 8^{+-}$ $\left.\mathrm{CD} 28^{+}, 5 \cdot 0(\mathrm{SEM} 0.1) \%, n 40\right)$ or $\mathrm{CD}^{+}$and $\mathrm{CD} 8^{+}$that expressed CD152 (CD4 ${ }^{+} \mathrm{CD} 8^{-} \mathrm{CD} 152^{+}, 0.12$ (SEM 0.01) \%, $n$ 40; $\mathrm{CD} 4{ }^{-} \mathrm{CD} 8^{+} \mathrm{CD} 152^{+}$; $1 \cdot 17$ (SEM 0.12) $\%, n$ 40) between obese and lean rats. However, splenocytes from obese rats did 
Table 3. The effect of dietary vaccenic acid (VA) on isolated mesenteric lymph node phospholipid fatty acid composition in JCR:LA/cp rats (Mean values with their standard errors for ten rats per group)

\begin{tabular}{|c|c|c|c|c|c|c|c|c|c|c|c|}
\hline \multirow[b]{3}{*}{ Fatty acids (g/100 g total fatty acids) } & \multicolumn{4}{|c|}{ Lean animals } & \multicolumn{4}{|c|}{ Obese animals } & & & \\
\hline & \multicolumn{2}{|c|}{ VA diet } & \multicolumn{2}{|c|}{ Control diet } & \multicolumn{2}{|c|}{ VA diet } & \multicolumn{2}{|c|}{ Control diet } & \multicolumn{3}{|c|}{ Significance* } \\
\hline & Mean & SEM & Mean & SEM & Mean & SEM & Mean & SEM & Diet & Genotype & Diet $\times$ genotype \\
\hline $14: 0$ & $1 \cdot 1$ & 0.2 & 0.5 & 0.2 & $1 \cdot 1$ & 0.2 & 1.0 & 0.3 & NS & NS & NS \\
\hline $16: 0$ & $21 \cdot 4^{\mathrm{C}}$ & 0.9 & $20 \cdot 2^{c}$ & 0.6 & $27 \cdot 7^{\mathrm{a}}$ & 0.3 & $25 \cdot 3^{b}$ & 0.8 & 0.01 & $<0.0001$ & NS \\
\hline $16: 1$ (cis-9) & $1 \cdot 0$ & $0 \cdot 2$ & 0.6 & 0.1 & 1.6 & 0.1 & 1.8 & 0.3 & NS & $<0.0001$ & NS \\
\hline $18: 0$ & $19 \cdot 1$ & $1 \cdot 1$ & $20 \cdot 0$ & 0.6 & $18 \cdot 6$ & 0.4 & $19 \cdot 2$ & 0.7 & NS & NS & NS \\
\hline VA (trans-11) & $1.9^{a}$ & 0.3 & $0 \cdot 1^{\mathrm{c}}$ & 0.04 & $0.7^{b}$ & 0.1 & $0.1^{\mathrm{c}}$ & 0.03 & $<0.0001$ & 0.003 & 0.002 \\
\hline $18: 1$ (cis-9) & $15 \cdot 4^{b}$ & $1 \cdot 1$ & $19 \cdot 6^{a}$ & $1 \cdot 2$ & $12 \cdot 8^{c}$ & 0.5 & $14 \cdot 6^{\mathrm{b}, \mathrm{c}}$ & 0.6 & 0.001 & $<0.0001$ & NS \\
\hline 18: 1 (cis-11) & 2.5 & 0.4 & 1.6 & 0.6 & 3.5 & 0.1 & 3.5 & 0.1 & NS & 0.01 & NS \\
\hline $18: 2 n-6$ & 19.5 & $1 \cdot 3$ & $17 \cdot 8$ & 0.6 & $12 \cdot 0$ & 0.2 & $11 \cdot 8$ & 0.3 & NS & $<0.0001$ & NS \\
\hline $18: 3 n-3$ & 0.7 & 0.1 & 0.9 & 0.1 & 0.7 & 0.1 & 0.9 & 0.1 & NS & NS & NS \\
\hline $20: 1 n-9$ & 0.6 & 0.1 & 0.5 & 0.1 & 0.2 & 0.04 & 0.2 & 0.04 & NS & $<0.0001$ & NS \\
\hline CLA (cis-9, trans-11) & 0.3 & 0.1 & ND & - & 0.2 & 0.1 & ND & - & 0.05 & NS & NS \\
\hline $20: 2 n-6$ & $1 \cdot 2$ & 0.1 & 1.0 & 0.1 & $1 \cdot 2$ & 0.1 & $1 \cdot 1$ & 0.1 & NS & NS & NS \\
\hline $20: 3 n-3$ & 0.6 & 0.1 & 0.6 & 0.1 & $1 \cdot 6$ & 0.1 & $1 \cdot 6$ & 0.1 & NS & $<0.0001$ & NS \\
\hline $20: 4 n-6$ & $13 \cdot 3$ & 0.9 & $14 \cdot 6$ & $1 \cdot 1$ & $16 \cdot 4$ & 0.5 & $16 \cdot 9$ & 0.7 & NS & 0.002 & NS \\
\hline $24: 1 n-9$ & 0.9 & 0.1 & $1 \cdot 0$ & 0.1 & 0.9 & 0.03 & $1 \cdot 0$ & 0.04 & NS & NS & NS \\
\hline $22: 5 n-3$ & 0.3 & 0.02 & 0.3 & 0.03 & 0.5 & 0.04 & 0.4 & 0.02 & NS & $<0.0001$ & NS \\
\hline $22: 6 n-3$ & 0.3 & 0.02 & 0.4 & 0.03 & 0.6 & 0.03 & 0.5 & 0.04 & NS & $<0.0001$ & 0.04 \\
\hline$\Sigma$ SFA & 41.5 & 1.8 & $40 \cdot 8$ & 0.8 & $47 \cdot 4$ & 0.4 & $45 \cdot 5$ & $1 \cdot 2$ & NS & $<0.0001$ & NS \\
\hline$\Sigma$ MFA & $22 \cdot 3$ & $1 \cdot 2$ & 23.5 & 0.6 & $19 \cdot 4$ & 0.7 & $21 \cdot 2$ & 0.8 & NS & 0.004 & NS \\
\hline$\Sigma$ PUFA & $36 \cdot 2$ & 1.0 & $35 \cdot 7$ & 1.0 & $33 \cdot 2$ & 0.4 & $33 \cdot 3$ & 1.0 & NS & 0.004 & NS \\
\hline$\sum n-6$ & $34 \cdot 0$ & $1 \cdot 0$ & 33.5 & 0.8 & $29 \cdot 6$ & 0.5 & $29 \cdot 8$ & 0.8 & NS & $<0.0001$ & NS \\
\hline$\sum n-3$ & 1.7 & 0.1 & 1.9 & 0.1 & 2.9 & 0.1 & $2 \cdot 7$ & 0.1 & NS & $<0.0001$ & NS \\
\hline PUFA:SFA ratio & 0.9 & 0.1 & 0.9 & 0.04 & 0.7 & 0.01 & 0.7 & 0.04 & NS & 0.0002 & NS \\
\hline$n-6: n-3$ ratio & $19 \cdot 5^{a}$ & 0.4 & $17 \cdot 8^{b}$ & 0.5 & $10 \cdot 4^{c}$ & 0.3 & $11 \cdot 1^{c}$ & 0.2 & NS & $<0.0001$ & 0.003 \\
\hline
\end{tabular}

NS, $P>0.05$; CLA, conjugated linoleic acid; ND, not detectable.

a,b,c Mean values within a row with unlike superscript letters were significantly different $(P<0.05$; LSMeans).

* Significant main effects (diet and genotype) were determined by two-way ANOVA.

have a 22 and $24 \%$ higher proportion of splenic $\mathrm{CD}^{+}$and $\mathrm{CD}^{+}$cells that expressed the IL-2 receptor $\left(\mathrm{CD} 25^{+}\right)$, respectively (Table 4). Similarly, there was an $11 \%$ higher proportion of $\mathrm{CD}^{+}$cells expressing CD25 in MLN cells, but unlike the spleen there was no difference in the proportion of $\mathrm{CD}^{+}$cells expressing CD25 in obese compared with lean rats (Table 5).
Notably, there was no effect of obesity on the intensity of CD4 staining in the spleen, but obese rats had a $132 \%$ higher proportion of CD4lo MLN cells compared with lean rats (Table 5).

Effect of diet. There was no effect of VA on the proportion of $\mathrm{CD}^{+}$and $\mathrm{CD}^{+}$cells expressing CD25, CD28 or

Table 4. Splenic immune cell phenotypes

(Mean values with their standard errors for ten rats per group)

\begin{tabular}{|c|c|c|c|c|c|c|c|c|c|c|c|}
\hline & \multicolumn{4}{|c|}{ Lean animals } & \multicolumn{4}{|c|}{ Obese animals } & & & \\
\hline & \multicolumn{2}{|c|}{ VA diet } & \multicolumn{2}{|c|}{ Control diet } & \multicolumn{2}{|c|}{ VA diet } & \multicolumn{2}{|c|}{ Control diet } & \multicolumn{3}{|c|}{ Significance* $^{\star}$} \\
\hline & Mean & SEM & Mean & SEM & Mean & SEM & Mean & SEM & Diet & Genotype & Diet $\times$ genotype \\
\hline $\mathrm{CD}^{+}(\%$ of cells $)$ & $46 \cdot 0$ & $1 \cdot 0$ & $48 \cdot 4$ & 1.4 & $50 \cdot 0$ & 0.7 & $49 \cdot 6$ & 1.0 & NS & 0.02 & NS \\
\hline $\mathrm{CD}^{+} \mathrm{CD}^{+} 0^{+}\left(\%\right.$ of $\left.\mathrm{CD}^{+}\right)$ & $27 \cdot 6$ & 0.5 & $26 \cdot 0$ & 0.7 & $26 \cdot 2$ & 0.6 & $27 \cdot 1$ & 0.6 & NS & NS & 0.03 \\
\hline $\mathrm{CD}^{+} \mathrm{CD}^{+}(\%$ of cells $)$ & $30 \cdot 7^{b}$ & 0.5 & $34 \cdot 3^{a}$ & 1.3 & $34 \cdot 4^{\mathrm{a}}$ & 0.6 & $34 \cdot 7^{\mathrm{a}}$ & 0.9 & 0.03 & 0.02 & NS \\
\hline $\mathrm{CD} 4 \mathrm{RC}^{+}\left(\%\right.$ of $\left.\mathrm{CD}^{+} \mathrm{CD}^{+}\right)$ & $10 \cdot 8$ & 0.4 & $16 \cdot 5$ & $1 \cdot 2$ & $11 \cdot 7$ & 0.5 & $19 \cdot 0$ & $2 \cdot 2$ & $<0.0001$ & NS & NS \\
\hline $\mathrm{CD}_{25}{ }^{+}\left(\%\right.$ of $\left.\mathrm{CD} 4^{+}\right)$ & $17 \cdot 8$ & 0.5 & $19 \cdot 2$ & 1.8 & 21.5 & 0.4 & 23.7 & 1.5 & NS & 0.0009 & NS \\
\hline $\mathrm{CD}^{+} \mathrm{CD}^{+}(\%$ of cells $)$ & 9.9 & 0.2 & $9 \cdot 0$ & 0.7 & $10 \cdot 0$ & 0.3 & $11 \cdot 0$ & 0.3 & NS & 0.02 & NS \\
\hline $\mathrm{CD}^{2} 5^{+}\left(\%\right.$ of $\left.\mathrm{CD}^{+}\right)$ & $7 \cdot 8$ & 0.3 & $8 \cdot 2$ & 0.5 & $9 \cdot 9$ & 0.7 & $9 \cdot 9$ & 0.6 & NS & 0.002 & NS \\
\hline $\mathrm{CD}^{+}{ }^{\mathrm{CD}} 4^{+}: \mathrm{CD}^{+}{ }^{+} \mathrm{CD} 8^{+}$ & $3 \cdot 1$ & 0.1 & 3.4 & 0.2 & 3.5 & 0.2 & $3 \cdot 2$ & 0.1 & NS & NS & NS \\
\hline $\mathrm{OX} 12^{+}(\%$ of cells $)$ & $32 \cdot 1$ & $2 \cdot 0$ & $29 \cdot 3$ & $2 \cdot 8$ & $29 \cdot 3$ & $1 \cdot 3$ & $29 \cdot 0$ & $2 \cdot 8$ & NS & NS & NS \\
\hline CD11b/c ${ }^{+}(\%$ of cells $)$ & $12 \cdot 2$ & 0.7 & $10 \cdot 9$ & 0.3 & $13 \cdot 3$ & 0.4 & $14 \cdot 6$ & $1 \cdot 1$ & NS & 0.002 & NS \\
\hline
\end{tabular}

VA, vaccenic acid; NS, $P>0.05$

a,b Mean values within a row with unlike superscript letters were significantly different $(P<0.05$; LSMeans).

* Significant main effects (diet and genotype) were determined by two-way ANOVA. 
Table 5. Mesenteric lymph node immune cell phenotypes

(Mean values with their standard errors for ten rats per group)

\begin{tabular}{|c|c|c|c|c|c|c|c|c|c|c|}
\hline & \multicolumn{4}{|c|}{ Lean animals } & \multicolumn{4}{|c|}{ Obese animals } & & \\
\hline & \multicolumn{2}{|c|}{ VA diet } & \multicolumn{2}{|c|}{ Control diet } & \multicolumn{2}{|c|}{ VA diet } & \multicolumn{2}{|c|}{ Control diet } & \multicolumn{2}{|c|}{ Significance* } \\
\hline & Mean & SEM & Mean & SEM & Mean & SEM & Mean & SEM & Diet & Genotype \\
\hline $\mathrm{CD}^{+}(\%$ of cells $)$ & $64 \cdot 6$ & $1 \cdot 6$ & 67.5 & $1 \cdot 3$ & $62 \cdot 7$ & $1 \cdot 3$ & $63 \cdot 2$ & 1.7 & NS & 0.04 \\
\hline $\mathrm{CD}^{+}{ }^{+} \mathrm{CD} 90^{+}\left(\%\right.$ of $\left.\mathrm{CD}^{+}\right)$ & $25 \cdot 2$ & 0.6 & 25.5 & 0.7 & $23 \cdot 7$ & 0.6 & 23.5 & 0.7 & NS & 0.01 \\
\hline $\mathrm{CD}^{+}{ }^{+} D 4^{+}(\%$ of cells $)$ & $47 \cdot 4^{\mathrm{b}}$ & 1.4 & $52 \cdot 7^{\mathrm{a}}$ & $1 \cdot 7$ & $47 \cdot 6^{\mathrm{b}}$ & $1 \cdot 0$ & $49 \cdot 7^{\mathrm{a}, \mathrm{b}}$ & 1.9 & 0.02 & NS \\
\hline CD4hi (\% of cells) & $42 \cdot 0$ & 1.5 & $45 \cdot 3$ & 3.5 & $32 \cdot 1$ & $2 \cdot 7$ & $35 \cdot 2$ & $4 \cdot 6$ & NS & 0.005 \\
\hline CD4lo (\% of cells) & $6 \cdot 2$ & $1 \cdot 3$ & 8.6 & 3.5 & $16 \cdot 9$ & $3 \cdot 3$ & $15 \cdot 9$ & 4.6 & NS & 0.002 \\
\hline $\mathrm{CD} 45 \mathrm{RC}^{+}\left(\%\right.$ of $\left.\mathrm{CD}^{+}\right)$ & $2 \cdot 2^{\mathrm{b}}$ & 0.1 & $2 \cdot 7^{\mathrm{b}}$ & 0.4 & $2 \cdot 7^{b}$ & 0.1 & $3 \cdot 7^{a}$ & 0.4 & 0.02 & 0.009 \\
\hline $\mathrm{CD}^{2} 5^{+}\left(\%\right.$ of $\left.\mathrm{CD} 4^{+}\right)$ & $16 \cdot 2$ & 0.3 & $18 \cdot 2$ & $1 \cdot 1$ & $19 \cdot 9$ & 0.7 & $18 \cdot 3$ & 1.3 & NS & 0.04 \\
\hline $\mathrm{CD}^{+} \mathrm{CD}^{+}(\%$ of cells $)$ & $11 \cdot 3$ & 0.8 & $10 \cdot 5$ & 0.3 & 8.7 & 0.6 & $9 \cdot 1$ & 0.6 & NS & 0.001 \\
\hline $\mathrm{CD}^{2} 5^{+}\left(\%\right.$ of $\left.\mathrm{CD}^{+}\right)$ & $10 \cdot 7$ & 0.3 & $10 \cdot 2$ & 0.7 & $10 \cdot 4$ & 0.5 & 8.6 & 0.8 & NS & NS \\
\hline $\mathrm{CD}^{+} \mathrm{CD}^{+}: \mathrm{CD}^{+} \mathrm{CD}^{+}$ & $4 \cdot 0^{\mathrm{b}}$ & 0.2 & $5 \cdot 1^{a}$ & 0.3 & $5 \cdot 7^{\mathrm{a}}$ & 0.4 & $5 \cdot 7^{\mathrm{a}}$ & 0.5 & NS & 0.002 \\
\hline $\mathrm{OX} 12^{+}(\%$ of cells $)$ & $18 \cdot 5$ & $1 \cdot 3$ & $22 \cdot 8$ & $1 \cdot 2$ & $18 \cdot 9$ & 0.8 & $18 \cdot 4$ & 1.9 & NS & NS \\
\hline CD $11 \mathrm{~b} / \mathrm{c}^{+}$(\% of cells) & 4.0 & 0.3 & $4 \cdot 2$ & 0.3 & 3.8 & 0.3 & 3.7 & 0.3 & NS & NS \\
\hline
\end{tabular}

VA, vaccenic acid; NS, $P>0.05$.

a,b Mean values within a row with unlike superscript letters were significantly different $(P<0.05$; LSMeans).

CD152 in both the spleen and MLN. There was also no diet effect on the intensity of the CD4 staining in the MLN cells (Table 5) and splenocytes.

Diet $\times$ genotype effect. There was no interaction between diet and genotype on immune cell activation markers.

\section{Immune cell maturation markers}

Effect of obesity. There was no effect of obesity on the proportion of splenic $\mathrm{T}$-cells that were new $\left(\mathrm{CD}^{+} \mathrm{CD}^{+} 0^{+}\right)$, and helper $\mathrm{T}$-cells that were naive $\left(\mathrm{CD} 45 \mathrm{RC}^{+}\right)$(Table 4). However, obese rats had a $7 \%$ lower proportion of MLN T-cells that were new (Table 5) and a $33 \%$ higher proportion of MLN helper T-cells that were naive $\left(\mathrm{CD} 4{ }^{+} \mathrm{CD} 45 \mathrm{RC}^{+}\right)$compared with lean rats.

Effect of diet. VA had no effect on the proportion of splenic and MLN T-cells that were new; however, VA-fed rats had 35-38\% lower proportions of splenic helper T-cells that were naive compared with rats fed the control diet (Table 4).

Effect of vaccenic acid in lean $\mathrm{v}$. obese rats. VA normalised the proportion of MLN helper T-cells that were naive in obese rats to proportions similar to those seen in lean rats (Table 5).

Diet $\times$ genotype effect. There was no interaction between diet and genotype on immune cell maturation markers in the MLN or on the proportion of splenic helper T-cells that were naive. There was a significant interaction in the proportion of splenic T-cells that were new; however, no significant differences between means were found by LSMeans.

\section{Ex vivo cytokine production}

Effect of obesity. Unstimulated MLN from obese rats produced higher concentrations of IL-10 compared with lean rats (Table 6). Stimulation of splenocytes with the T-cell mitogen ConA resulted in a $43 \%$ higher IL- 6 concentration in supernatant fractions from obese compared with lean rats, but there was no difference between groups in the other cytokines measured (Table 6). Despite the lower proportion of MLN T-cells from obese rats, they produced 39-81\% higher concentrations of IL-2, IL-10, IFN $\gamma$ and TNF $\alpha$ when stimulated with ConA compared with leans (Table 6). Although there was a higher proportion of splenic monocytes/macrophages, there was only a trend $(P=0.0518)$ towards a higher concentration of TNF $\alpha$ produced with LPS stimulation (Table 6).

Effect of diet. Splenocytes from VA-fed rats produced $16-23 \%$ lower concentrations of IL-2, IL-10 and TNF $\alpha$, with no difference in the concentration of IFN $\gamma$ and IL-6 after ConA stimulation (Table 6). VA had no effect on the concentration of IL- $1 \beta$, IL- 6 and $\mathrm{TNF} \alpha$ produced in response to LPS, but there was a $43 \%$ lower concentration of IL-10 produced.

Effect of vaccenic acid in lean $\mathrm{v}$. obese rats. Unstimulated cells from lean rats fed VA produced lower concentrations of IL-10 compared with those from lean rats fed the control diet, but there was no difference between dietary treatments in the obese rats. VA had no effect on cytokine concentrations produced by splenic and MLN cells after mitogenic stimulation from lean rats, except for a lower concentration of IL-10 by splenocytes stimulated with LPS (Table 6). However, obese rats fed VA produced $32 \%$ less TNF $\alpha$ from ConA-stimulated splenocytes compared with obese rats fed the control diet. In addition, obese rats fed VA produced 34-38\% lower concentrations of IL-2 and TNF $\alpha$ (similar to concentrations produced by lean controls) compared with obese rats fed the control diet. Obese rats fed VA also produced 35-136\% higher concentrations of IL-10 and IFN $\gamma$ compared with the other groups (Table 6).

Diet $\times$ genotype effect. There was no interaction between diet and genotype on ex vivo cytokine production.

\section{Discussion}

There is increasing evidence that obesity is a chronic inflammatory state with impaired immune responses ${ }^{(12,13)}$. The JCR:LA- $c p$ rat has been shown to have higher circulating 
Table 6. Cytokine production $(\mathrm{pg} / \mathrm{ml})$ by spleen and mesenteric lymph node $(\mathrm{MLN})$ cells stimulated with concanavalin $A$ (ConA) or lipopolysaccharide (LPS) for $48 \mathrm{~h}$

(Mean values with their standard errors for nine or ten rats per group)

\begin{tabular}{|c|c|c|c|c|c|c|c|c|c|c|}
\hline & \multicolumn{4}{|c|}{ Lean animals } & \multicolumn{4}{|c|}{ Obese animals } & & \\
\hline & \multicolumn{2}{|c|}{ VA diet } & \multicolumn{2}{|c|}{ Control diet } & \multicolumn{2}{|c|}{ VA diet } & \multicolumn{2}{|c|}{ Control diet } & \multicolumn{2}{|c|}{ Significance* } \\
\hline & Mean & SEM & Mean & SEM & Mean & SEM & Mean & SEM & Diet & Genotype \\
\hline \multicolumn{11}{|l|}{ Unstimulated } \\
\hline IL-10 (spleen) & $220^{\mathrm{b}}$ & 10 & $434^{\mathrm{a}}$ & 79 & $258^{a, b}$ & $32^{b}$ & $350^{\mathrm{a}}$ & 91 & 0.04 & NS \\
\hline IL-10 (MLN) & 34 & 4 & 39 & 4 & 48 & 5 & 51 & 4 & NS & 0.004 \\
\hline \multicolumn{11}{|c|}{ Splenocytes ConA } \\
\hline IL-2 & 2313 & 176 & 2662 & 202 & 2264 & 150 & 2759 & 242 & 0.04 & NS \\
\hline IL-10 & 393 & 52 & 480 & 53 & 402 & 54 & 537 & 59 & 0.03 & NS \\
\hline $\mathrm{TNF} \alpha$ & $186^{\mathrm{b}, \mathrm{c}}$ & 16 & $218^{a, b}$ & 18 & $167^{\mathrm{c}}$ & $5 \cdot 1$ & $244^{a}$ & 29 & 0.01 & NS \\
\hline $\mathrm{IFN} \gamma$ & 1296 & 109 & 1025 & 208 & 1112 & 181 & 783 & 173 & NS & NS \\
\hline IL-6 & 199 & 23 & 155 & 28 & 210 & 38 & 300 & 53 & NS & 0.04 \\
\hline \multicolumn{11}{|l|}{ MLN ConA } \\
\hline IL-2 & $232^{\mathrm{b}}$ & 29 & $297^{b}$ & 54 & $299^{b}$ & 24 & $486^{a}$ & 74 & 0.02 & 0.002 \\
\hline IL-10 & $151^{\mathrm{b}}$ & 27 & $94^{\mathrm{b}}$ & 30 & $207^{\mathrm{a}}$ & 28 & $153^{b}$ & 23 & 0.02 & 0.008 \\
\hline $\mathrm{TNF} \alpha$ & $44 \cdot 0^{\mathrm{b}}$ & 7.5 & $59 \cdot 0^{\mathrm{b}}$ & $10 \cdot 1$ & $58 \cdot 8^{\mathrm{b}}$ & $7 \cdot 0$ & $88.5^{\mathrm{a}}$ & 11.9 & 0.03 & 0.03 \\
\hline $\mathrm{IFN} \gamma$ & $181^{\mathrm{b}}$ & 34 & $157^{b}$ & 44 & $371^{\mathrm{a}}$ & 22 & $242^{b}$ & 37 & NS & 0.001 \\
\hline \multicolumn{11}{|l|}{ Splenocytes LPS } \\
\hline IL-1 $\beta$ & 107 & 20 & 137 & 17 & 95 & 12 & 89 & 15 & NS & NS \\
\hline IL-6 & 382 & 37 & 367 & 45 & 409 & 33 & 326 & 45 & NS & NS \\
\hline IL-10 & $613^{\mathrm{b}}$ & 35 & $1257^{a}$ & 274 & $799^{a, b}$ & 27 & $1229^{a}$ & $215^{\mathrm{a}}$ & 0.001 & NS \\
\hline $\mathrm{TNF} \alpha$ & 662 & 57 & 567 & 37 & 728 & 35 & 645 & 51 & NS & NS \\
\hline
\end{tabular}

VA, vaccenic acid; NS, $P>0.05$; IFN, interferon.

a,b,c Mean values within a row with unlike superscript letters were significantly different $(P<0.05$; LSMeans).

* Significant main effects (diet and genotype) were determined by two-way ANOVA. There were no significant interactions between diet and genotype.

levels of haptoglobin ${ }^{(11)}$ (a systemic marker of inflammation) and we report here that abnormalities in immune function exist (Fig. 1). MLN cells of obese rats produced higher concentrations of IL-2, IL-10, IFN $\gamma$ and TNF $\alpha$ in response to mitogenic challenge (Table 6), indicating a pro-inflammatory tendency. This observation is consistent with other reports of splenocytes from fa/fa Zucker rats ${ }^{(12)}$ and peripheral blood mononuclear cells from humans with high $\mathrm{BMI}^{(21)}$. Inflammation involves activated T-cells ${ }^{(22)}$. An indicator of T-cell activation that has not been measured in other models of obesity is the down-modulation of CD4, indicated by a lower cell surface expression of $\mathrm{CD} 4{ }^{(23)}$. Obese rats from the present study had a higher proportion of MLN CD4lo cells (Table 5), suggesting a higher proportion of activated cells, which is supported by a higher concentration of IL-10 produced by unstimulated MLN cells relative to lean rats. A higher production of cytokines by stimulated MLN cells (Table 6) is also consistent with a higher proportion of activated T-cells.

Experiments using $f a / f a$ Zucker rats ${ }^{(24)}$ and obese human subjects ${ }^{(25)}$ have indicated a lower proliferative response of lymphocytes when stimulated ex vivo. The present results are not consistent with other rodent models of obesity that have shown lower proportions of $\mathrm{CD}^{+} \mathrm{CD} 25^{+}$splenic cells ${ }^{(12)}, \mathrm{CD}^{+}{ }^{+} \mathrm{CD} 25^{+}$MLN cells ${ }^{(26)}$ and IL-2 production after mitogenic stimulation ${ }^{(12,27)}$. The higher proportion of MLN helper T-cells that were $\mathrm{CD} 25^{+}$in combination with the higher IL-2 production in response to ConA suggest that MLN cells from the obese JCR:LA- $c p$ rats may have a higher proliferative response compared with lean rats. It is unclear whether the differences among rodent models are due to environmental differences (i. e. housing or diet), genetic differences or experimental conditions (i.e. age), all of which influence immune function. Social isolation of male rats has been shown to be immunosuppressive ${ }^{(28)}$; however, because all the rats in the present study were housed in the same manner, any effects of individual housing on immune function would be the same between dietary treatment groups and between genotypes.

CD45RC expression has been used to identify naive $\left(\mathrm{CD}^{2} 5 \mathrm{RC}^{+}\right) v$. memory T-cells $\left(\mathrm{CD} 45 \mathrm{RC}^{-}\right)^{(29)}$ as well as T-helper $(\mathrm{Th}) 1\left(\mathrm{CD} 45 \mathrm{RC}^{+}\right) v$. Th2 $\left(\mathrm{CD}^{2} 5 \mathrm{RC}^{-}\right)^{(30,31)}$ in rats. Obese JCR:LA- $c p$ rats had a higher proportion of MLN helper T-cells that were $\mathrm{CD} 45 \mathrm{RC}^{+}$compared with lean rats, while the VA-fed rats had lower proportions of splenic and MLN helper T-cells that were CD45RC ${ }^{+}$compared with controls (Table 5); however, the concentrations of cytokines produced were not consistent with a greater proportion of naive or Th1 cells in obese MLN or lower proportion of splenic Th1 cells with dietary VA.

This is the first study to report a lower proportion of new MLN T-cells in an obese animal model (Table 5). It has been hypothesised that, over time, fewer new T-cells would negatively affect the $\mathrm{T}$-cell repertoire, leaving the rat more susceptible to disease ${ }^{(32)}$, which is consistent with the reduced wound healing in the JCR:LA- $c p$ rat $^{(33)}$ and the observation that human obesity is associated with an increased risk of infection (for a review, see Falagas \& Kompoti $^{(13)}$ ). Previous reports in fa/fa Zucker rats ${ }^{(12,24)}$, diet-induced obese mice ${ }^{(26)}$ and humans ${ }^{(21)}$ have all described T-cell lymphopenia in the obese state. Consistent with these studies, the obese JCR:LA- $c p$ rat had a lower proportion of MLN T-cells 
Effects of obesity on immune parameters

(A) Spleen

Spleen weight perg of body weight $\downarrow$

No. of splenocytes per g of spleen $\downarrow$

\% T-cells $\left(\mathrm{CD}^{+}\right) \uparrow$

$\%$ Helper T-cells $\left(\mathrm{CD}^{+} \mathrm{CD}^{+}\right) \uparrow$

\% Cytotoxic T-cells $\left(\mathrm{CD}^{+}{ }^{+} \mathrm{CD} 8^{+}\right) \uparrow$

$\%$ macrophage/monocytes $\left(\mathrm{CD} 11 \mathrm{~b} / \mathrm{c}^{+}\right) \uparrow$

$\% \mathrm{CD}^{2} 5^{+}$helper T $\uparrow$

\% CD25+ cytotoxic T $\uparrow$

IL-6 $\uparrow$ after stimulation with ConA
(B) Mesenteric lymph nodes

$\%$ T-cells $\left(\mathrm{CD}^{+}\right) \downarrow$
$\%$ New T-cells $\left(\mathrm{CD} 3^{+} \mathrm{CD} 90^{+}\right) \downarrow$
$\%$ Cytotoxic T-cells $\left(\mathrm{CD}^{+} \mathrm{CD}^{+}\right) \downarrow$
$\%$ Naive $\left(\mathrm{CD} 4^{+} \mathrm{CD} 45 \mathrm{RC} \mathrm{C}^{+}\right) \uparrow$
$\%$ CD $25^{+}$helper $\mathrm{\uparrow}$
$\% \mathrm{CD} 4 \mathrm{lo} \uparrow$
IL-2 $\uparrow$ after stimulation with ConA
IL-10 $\uparrow$
IFN $\gamma \uparrow$
TNF $\alpha \uparrow$

(C) Spleen

Spleen weight per g of body weight $\uparrow$ in leans rats

No. of splenocytes per spleen $\downarrow$

$\%$ Helper T-cells $\left(\mathrm{CD} 3^{+} \mathrm{CD} 4^{+}\right) \downarrow$ in lean rats

$\%$ Naive $\left(\mathrm{CD}^{+}{ }^{+} \mathrm{CD} 45 \mathrm{RC} \mathrm{C}^{+}\right) \downarrow$

$\mathrm{IL}-2 \downarrow$

IL-10 $\downarrow$

$\mathrm{TNF} \alpha \downarrow$
(D) Mesenteric lymph nodes

$\%$ Helper T-cells $\left(\mathrm{CD}^{+} \mathrm{CD} 4^{+}\right) \downarrow$ in lean rats

\% Naive $\left(\mathrm{CD}^{+}{ }^{+} \mathrm{CD} 45 \mathrm{RC}^{+}\right) \downarrow$

Normalisation of IL-2 and TNF $\alpha$ in obese rats

IL-10个 in obese rats

Fig. 1. Summary of the effects of obesity $(A)$ and $(B)$ and vaccenic acid (VA) (C) and (D) on immune cell phenotypes and cytokine production in the spleen $(A)$ and $(C)$ and mesenteric lymph nodes $(B)$ and $(D)$ of JCR:LA/cp rats. ConA, concanavalin A; IFN, interferon.

(Table 5). However, in contrast with these reports, the obese JCR:LA- $c p$ rat had a higher proportion of splenic T-cells (Table 5). This difference in the effect of obesity on the proportion of T-cells between organs could be due to the adipose tissue that surrounds the MLN, but not the spleen, a T-cell homing defect or an altered proportion of an unidentified population of MLN cells in the obese rats (i.e. dendritic cells).

MLN cells from obese rats produced higher concentrations of all cytokines measured (despite a lower proportion of T-cells), while there was no effect of obesity on the concentration of the cytokines produced by splenocytes with the exception of IL-6 in response to ConA (Fig. 1). A possible explanation for the differences in immune function between organs is that the MLN are embedded in visceral fat, while the spleen is not. Visceral fat produces adipokines which have various effects on the immune system (for a review, see Lago et al. $\left.{ }^{(34)}\right)$. In the diet-induced obese mouse, visceral fat accumulation was linked with lymph node atrophy and lymphocyte apoptosis ${ }^{(26)}$. Interestingly, CLA feeding in the $\mathrm{fa} / \mathrm{fa}$ rat has been shown to alter concentrations of adipokines in both serum and adipose tissue ${ }^{(35)}$. Our group has shown that VA-fed rats have a higher proportion of CLA within TAG isolated from adipose tissue relative to control-fed rats ${ }^{(11)}$. Adipokine concentrations in serum and adipose tissue were not measured in the present study; therefore, it is unknown whether MLN and spleen cells were exposed to different concentrations of adipokines. Although the function of the immune cells was assessed ex vivo without visceral fat, chronic exposure to adipokines in vivo may have permanently 'programmed' the immune cells to respond in a particular manner even in the absence of the adipokine signal. 
Splenocytes from VA-fed rats produced lower concentrations of IL-10 when unstimulated, ConA-stimulated and LPS-stimulated compared with splenocytes from control-fed rats (Table 6). This is consistent with the lower serum IL-10 concentrations in obese rats fed VA in the study by Wang et al. ${ }^{(11)}$. However, there was no evidence of an increased splenic pro-inflammatory response with VA, indicating that the anti-inflammatory properties of IL-10 were not required to control inflammation. Conversely, stimulated MLN cells from VA-fed rats produced higher concentrations of IL-10 compared with control-fed rats (Table 6). It is likely that the inflammatory signals emanating from the adipose tissue affect immune cells in the resident MLN; therefore it would be beneficial to have more regulatory $\mathrm{T}$-cells producing higher concentrations of IL-10 in the MLN to regulate or control the locally stimulated immune response. In fact, we did observe a normalisation of IL- 2 and $\mathrm{TNF} \alpha$ production with a higher production of IL-10 in MLN with VA feeding in the obese rats (Table 6). For all the other cytokines measured, there was not an opposite effect of VA in splenocytes compared with MLN; however, there was less of an effect in the splenocytes compared with MLN.

The effect of VA on IL-10 production in the MLN might be mediated by its conversion into CLA (cis-9, trans-11). Previous reports have shown that CLA (cis-9, trans-11) can enhance IL-10 mRNA and protein concentrations produced by stimulated dendritic cells ${ }^{(36)}$. It is unclear whether CLA would have similar effects in different immune cell phenotypes. MLN contain mainly T-cells while the spleen contains a more even split between $\mathrm{T}$ - and B-cells. The differential effects of VA on the concentration of cytokines produced between splenocytes and MLN cells might be accounted for by the differences in the cell types present. To further support this hypothesis, after stimulation with the polyclonal T-cell mitogen, ConA, splenocytes from rats fed VA produced lower concentrations of IL-2 and TNF $\alpha$ compared with control-fed rats, but no difference in IL-2 or TNF $\alpha$ production was detected when splenocytes were stimulated with the B-cell/macrophage/dendritic cell mitogen LPS (Table 6). This suggests that feeding a diet enriched in VA may have altered T-cell function to a greater extent than that of antigen-presenting cells. There was no effect of diet on the proportion of splenic macrophages or B-cells; however, we did not measure the proportion of dendritic cells in this tissue.

In recent years, trans-fats have garnered interest due to concerns that dietary trans-fat increases the risk of CHD by favouring a pro-inflammatory state (for a review, see Mozaffarian $^{(37)}$ ). However, it stands to reason that individual trans-fatty acids may have different immunological effects. Plasma markers of inflammation were positively related to trans-oleic acid (mainly elaidic acid) ${ }^{(38)}$, while our group has reported that dietary VA did not increase systemic markers of inflammation (serum haptoglobin and IL-6 concentrations) in obese or lean rodents ${ }^{(11)}$. The present report is the first to examine the effects of VA on MLN and spleen cell immune function in the obese/insulin-resistant JCR:LA- $c p$ rat. While other reports have shown hydrogenated fat increased peripheral blood mononuclear cell inflammatory cytokine production $^{(39)}$, we report that feeding $1.5 \%$ (w/w) VA did not increase the inflammatory response of splenic and MLN cells in both healthy rodents and those with the metabolic syndrome, but favourably altered the pro-inflammatory tendency of MLN cells in obese JCR:LA-cp rats.

Previous studies have shown that dietary CLA (cis-9, trans-11) has immunomodulatory effects in disease states, with no alteration of immune function in the healthy state ${ }^{(12,40)}$. Therefore, we were not expecting to see an interaction between diet and genotype. Dietary VA did not alter the proportion of any of the immune cell phenotypes measured in lean rats, except for a lower proportion of helper T-cells. This decrease in helper T-cell proportions in VA-fed rats was associated with lower concentrations of cytokines produced after mitogenic stimulation; however, the physiological implications of these small changes are probably minor. Dietary VA did not normalise any of the altered immune cell phenotypes (including activation markers) in obese rats to levels similar to those seen in lean rats with the exception of the proportion

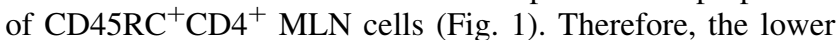
cytokine production by VA-fed rats after mitogenic stimulation (Table 6) cannot be explained by the proportion of activated cells in immune organs.

PPAR- $\alpha$ and PPAR- $\gamma$ are expressed by lymphocytes (for a review, see Glass \& Ogawa $\left.{ }^{(41)}\right)$. PPAR agonists, such as CLA (cis-9, trans-11), have been shown to inhibit inflammatory cytokine production $^{(42,43)}$; therefore, the lower inflammatory cytokine production with VA feeding may be due to its conversion to CLA (cis-9, trans-11). CLA (cis-9, trans-11) has shown little or non-significant effects on immune function in human studies ${ }^{(44-46)}$. However, it is important to note that most of the trials have been done in healthy adults where we would expect to see very little effects on immune function. We have shown that VA can have different effects on the systemic immune response compared with the mesenteric immune response (Fig. 1). The human trials were not able to access the MLN where we observed the 'normalisation' of cytokine production.

The VA diets contained $9 \%$ of total fatty acids as VA and $0 \%$ CLA (Table 1). It is generally accepted that plasma phospholipid fatty acid composition reflects dietary intake. Therefore, it is interesting to note that despite a similar dietary content of $16: 0,18: 2 n-6$ and $18: 0$ between the VA diet and control diet, VA-fed rats had higher concentrations of these fatty acids in plasma phospholipids compared with control-fed rats (Table 2). The plasma phospholipid concentration of VA in the lean VA-fed rats was similar to the dietary composition; however, the obese VA-fed rats had almost twice as much VA in plasma phospholipids compared with lean VA-fed rats (Table 2). This higher concentration of VA in plasma phospholipids in the obese state might be due to impaired incorporation of VA into tissues as evidenced by the lower concentration of VA in MLN phospholipids in the obese rats compared with lean VA-fed rats (Table 3). This lower concentration of VA in obese $v$. lean VA-fed rats is consistent with our report on VA proportions in adipose tissue $\mathrm{TAG}^{(11)}$. Similar to plasma phospholipids, the proportion of MLN phospholipid as 16:0 was also higher in obese compared with lean rats and with the VA compared with the control diets, which is consistent with a report showing a higher proportion of 16:0 in splenocyte phospholipids in Zucker rats fed CLA-containing diets ${ }^{(12)}$.

VA-fed rats had $0.7-1.9 \%$ of total MLN phospholipids as VA and $0.3 \%$ CLA (cis-9, trans-11). Although the MLN 
phospholipid concentration of CLA (cis-9, trans-11) is small in the VA-fed rats, it is similar to the proportion of CLA (cis-9, trans-11) in splenocyte phospholipids in the study by Ruth et al. ${ }^{(12)}$ who fed Zucker rats diets containing $5 \cdot 3 \%$ of total fatty acids as CLA (cis-9, trans-11). In that study, the cis-9, trans-11 isomer of CLA had beneficial effects on the proliferative and immunoregulatory response of T-cells in the Zucker rat ${ }^{(12)}$. In the present study where we fed VA without CLA (cis-9, trans-11), the effects we observed suggest that some of the beneficial effects of VA on immune function may be mediated by its conversion into CLA. This is the first study to report the effect of VA on MLN phospholipid fatty acid concentrations and there are currently no published reports on the effect of dietary CLA (cis-9, trans-11) on MLN phospholipid concentrations. In vitro studies have shown that other dietary fatty acids, in particular EPA, can interfere with the conversion of VA into CLA (cis-9, trans-11) ${ }^{(47)}$; however, our diets were designed to contain a balanced polyunsaturated: saturated ratio, not high in PUFA, with no EPA. Overall, we achieved a higher proportion of VA and CLA (cis-9, trans-11) in MLN phospholipids (Table 3) compared with the proportion of VA (0.45 (SEM 0.06)) and CLA (cis-9, trans-11) (0.16 (SEM 0.03)) in mononuclear cell lipids from healthy humans consuming a dietary concentration of VA similar to the one used in the present study (about $8 \%$ of dietary fat) for $42 \mathrm{~d}^{(14)}$. Both cell-culture studies ${ }^{(48)}$ and animal feeding trials ${ }^{(49)}$ have shown that VA is converted into CLA (cis-9, trans-11); however, the anti-inflammatory and anti-cancer effects were specific to CLA (cis-9, trans-11). With the present study design, we are not able to separate out the effects of VA from CLA (cis-9, trans-11) on immune function.

In summary, the obese JCR:LA-cp rat, similar to other models of obesity, demonstrates a pro-inflammatory tendency when stimulated ex vivo. The present study is the first to report that unlike other industrially produced transfats, the natural trans-fat VA does not promote an inflammatory response by MLN and splenic cells in lean or obese JCR:LA- $c p$ rats. Although the effects of dietary VA on immune cell phenotypes and function were small with few significant diet and genotype interactions, VA appears to favourably alter the pro-inflammatory tendency of MLN cells from obese JCR:LA-cp rats. The effects of VA on immune function in vivo on immunologically relevant markers of disease (i.e. arterial plaque formation) should be examined in future studies. In North America, CLA but not VA is excluded from the total trans content on packaged foods ${ }^{(50,51)}$, as there is insufficient evidence to conclude that VA does not negatively affect health. The present report provides evidence in an animal model that dietary VA at $3 \%$ of energy may provide some health benefits.

\section{Acknowledgements}

The present study was supported by operating grants from the Beef Information Centre to C. J. F. and S. D. P. and the Alberta Agriculture Funding Consortium to the Alberta CLA Network to C. J. F.
We wish to thank Susan Goruk, Kristina MacNaughton and Tara Martin for their technical assistance, and Sharon Sokolik for animal care.

H. J. B. analysed data derived from the various experimental procedures, performed statistical analysis, wrote the manuscript and received feedback from the co-authors. C. A. G. prepared experimental diets, and performed and coordinated all laboratory analysis. M. R. R., S. D. P. and C. J. F. provided intellectual guidance at all stages.

There are no conflicts of interest to declare.

\section{References}

1. Health Canada (2007) Trans fat. http://www.hc-sc.gc.ca/fn-an/ nutrition/gras-trans-fats/index_e.html (accessed 12 October 2007).

2. Institute of Medicine (2005) Dietary fats: total fat and fatty acids. In Dietary Reference Intakes for Energy, Carbohydrate, Fiber, Fat, Fatty Acids, Cholesterol, Protein, and Amino Acids, pp. 422-541. Washington, DC: The National Academies Press.

3. Eckel RH, Borra S, Lichtenstein AH, et al. (2007) Understanding the complexity of trans fatty acid reduction in the American diet: American Heart Association Trans Fat Conference 2006: report of the Trans Fat Conference Planning Group. Circulation 115, 2231-2246.

4. Lock AL \& Bauman DE (2004) Modifying milk fat composition of dairy cows to enhance fatty acids beneficial to human health. Lipids 39, 1197-1206.

5. Chardigny JM, Destaillats F, Malpuech-Brugere C, et al. (2008) Do trans fatty acids from industrially produced sources and from natural sources have the same effect on cardiovascular disease risk factors in healthy subjects? Results of the trans Fatty Acids Collaboration (TRANSFACT) study. Am J Clin Nutr 87, 558-566.

6. Bhattacharya A, Banu J, Rahman M, et al. (2006) Biological effects of conjugated linoleic acids in health and disease. J Nutr Biochem 17, 789-810.

7. Mir PS, McAllister TA, Scott S, et al. (2004) Conjugated linoleic acid-enriched beef production. Am J Clin Nutr 79, $1207 \mathrm{~S}-1211 \mathrm{~S}$

8. Cruz-Hernandez C, Kramer JK, Kennelly JJ, et al. (2007) Evaluating the conjugated linoleic acid and trans 18:1 isomers in milk fat of dairy cows fed increasing amounts of sunflower oil and a constant level of fish oil. J Dairy Sci 90, 3786-3801.

9. Gillis MH, Duckett SK \& Sackmann JR (2007) Effects of supplemental rumen-protected conjugated linoleic acid or corn oil on lipid content and palatability in beef cattle. J Anim Sci 85, 1504-1510.

10. Turpeinen AM, Mutanen M, Aro A, et al. (2002) Bioconversion of vaccenic acid to conjugated linoleic acid in humans. Am J Clin Nutr 76, 504-510.

11. Wang Y, Lu J, Ruth MR, et al. (2008) Trans-11 vaccenic acid dietary supplementation induces hypolipidemic effects in JCR:LA-cp rats. J Nutr 138, 2117-2122.

12. Ruth MR, Taylor CG, Zahradka P, et al. (2008) Abnormal immune responses in fa/fa Zucker rats and effects of feeding conjugated linoleic acid. Obesity (Silver Spring) 16, 1770-1779.

13. Falagas ME \& Kompoti M (2006) Obesity and infection. Lancet Infect Dis 6, 438-446.

14. Kuhnt K, Kraft J, Vogelsang H, et al. (2007) Dietary supplementation with trans-11- and trans-12-18:1 increases cis-9, trans-11-conjugated linoleic acid in human immune cells, but 
without effects on biomarkers of immune function and inflammation. Br J Nutr 97, 1196-1205.

15. Russell JC, Graham SE \& Richardson M (1998) Cardiovascular disease in the JCR:LA-cp rat. Mol Cell Biochem 188, 113-126.

16. Eckel RH, Grundy SM \& Zimmet PZ (2005) The metabolic syndrome. Lancet 365, 1415-1428.

17. Field CJ (1995) A diet producing a low diabetes incidence modifies immune abnormalities in diabetes-prone BB rats. $J$ Nutr 125, 2595-2603.

18. Field CJ, Ryan EA, Thomson AB, et al. (1988) Dietary fat and the diabetic state alter insulin binding and the fatty acyl composition of the adipocyte plasma membrane. Biochem $J \mathbf{2 5 3}$, 417-424.

19. Layne KS, Goh YK, Jumpsen JA, et al. (1996) Normal subjects consuming physiological levels of $18: 3(n-3)$ and $20: 5(n-3)$ from flaxseed or fish oils have characteristic differences in plasma lipid and lipoprotein fatty acid levels. J Nutr 126, 2130-2140.

20. Cruz-Hernandez C, Deng Z, Zhou J, et al. (2004) Methods for analysis of conjugated linoleic acids and trans-18:1 isomers in dairy fats by using a combination of gas chromatography, silver-ion thin-layer chromatography/gas chromatography, and silver-ion liquid chromatography. J AOAC Int 87, 545-562.

21. Tanaka S, Isoda F, Ishihara $\mathrm{Y}$, et al. (2001) T lymphopaenia in relation to body mass index and TNF- $\alpha$ in human obesity: adequate weight reduction can be corrective. Clin Endocrinol (Oxf) 54, 347-354.

22. Hansson GK, Robertson AK \& Soderberg-Naucler C (2006) Inflammation and atherosclerosis. Annu Rev Pathol 1, 297-329.

23. Marsh M \& Pelchen-Matthews A (1996) Endocytic and exocytic regulation of CD4 expression and function. Curr Top Microbiol Immunol 205, 107-135.

24. Tanaka S, Isoda F, Yamakawa T, et al. (1998) T lymphopenia in genetically obese rats. Clin Immunol Immunopathol 86, 219-225.

25. Nieman DC, Henson DA, Nehlsen-Cannarella SL, et al. (1999) Influence of obesity on immune function. $J$ Am Diet Assoc 99, 294-299.

26. Kim CS, Lee SC, Kim YM, et al. (2008) Visceral fat accumulation induced by a high-fat diet causes the atrophy of mesenteric lymph nodes in obese mice. Obesity (Silver Spring) 16, 1261-1269.

27. Mito N, Hosoda T, Kato C, et al. (2000) Change of cytokine balance in diet-induced obese mice. Metabolism 49 , $1295-1300$.

28. Jessop JJ \& Bayer BM (1989) Time-dependent effects of isolation on lymphocyte and adrenocortical activity. J Neuroimmunol 23, 143-147.

29. Bunce C \& Bell EB (1997) CD45RC isoforms define two types of CD4 memory $\mathrm{T}$ cells, one of which depends on persisting antigen. $J$ Exp Med 185, 767-776.

30. Subra JF, Cautain B, Xystrakis E, et al. (2001) The balance between CD45RChigh and CD45RClow CD4 T cells in rats is intrinsic to bone marrow-derived cells and is genetically controlled. J Immunol 166, 2944-2952.

31. McKnight AJ, Barclay AN \& Mason DW (1991) Molecular cloning of rat interleukin $4 \mathrm{cDNA}$ and analysis of the cytokine repertoire of subsets of $\mathrm{CD}^{+} \mathrm{T}$ cells. Eur J Immunol 21, $1187-1194$.

32. La Gruta NL, Driel IR \& Gleeson PA (2000) Peripheral T cell expansion in lymphopenic mice results in a restricted $\mathrm{T}$ cell repertoire. Eur J Immunol 30, 3380-3386.

33. Bauer BS, Ghahary A, Scott PG, et al. (2004) The JCR:LA-cp rat: a novel model for impaired wound healing. Wound Repair Regen 12, 86-92.
34. Lago F, Dieguez C, Gomez-Reino J, et al. (2007) Adipokines as emerging mediators of immune response and inflammation. Nat Clin Pract Rheumatol 3, 716-724.

35. Noto A, Zahradka P, Yurkova N, et al. (2007) Dietary conjugated linoleic acid decreases adipocyte size and favorably modifies adipokine status and insulin sensitivity in obese, insulin-resistant rats. Metabolism 56, 1601-1611.

36. Loscher CE, Draper E, Leavy O, et al. (2005) Conjugated linoleic acid suppresses NF- $\kappa \mathrm{B}$ activation and IL-12 production in dendritic cells through ERK-mediated IL-10 induction. J Immunol 175, 4990-4998.

37. Mozaffarian D (2006) Trans fatty acids - effects on systemic inflammation and endothelial function. Atheroscler Suppl 7, 29-32.

38. Lopez-Garcia E, Schulze MB, Meigs JB, et al. (2005) Consumption of trans fatty acids is related to plasma biomarkers of inflammation and endothelial dysfunction. $J$ Nutr 135, 562-566.

39. Han SN, Leka LS, Lichtenstein AH, et al. (2002) Effect of hydrogenated and saturated, relative to polyunsaturated, fat on immune and inflammatory responses of adults with moderate hypercholesterolemia. J Lipid Res 43, 445-452.

40. Turpeinen AM, Ylönen N, von Willebrand E, et al. (2008) Immunological and metabolic effects of cis-9 trans-11-conjugated linoleic acid in subjects with birch pollen allergy. $\mathrm{Br} J$ Nutr 100, 112-119.

41. Glass CK \& Ogawa S (2006) Combinatorial roles of nuclear receptors in inflammation and immunity. Nat Rev Immunol 6, 44-55.

42. Marx N, Kehrle B, Kohlhammer K, et al. (2002) PPAR activators as antiinflammatory mediators in human $\mathrm{T}$ lymphocytes: implications for atherosclerosis and transplantation-associated arteriosclerosis. Circ Res 90, 703-710.

43. Yu Y, Correll PH \& Vanden Heuvel JP (2002) Conjugated linoleic acid decreases production of pro-inflammatory products in macrophages: evidence for a PPAR $\gamma$-dependent mechanism. Biochim Biophys Acta 1581, 89-99.

44. Mullen A, Moloney F, Nugent AP, et al. (2007) Conjugated linoleic acid supplementation reduces peripheral blood mononuclear cell interleukin-2 production in healthy middle-aged males. J Nutr Biochem 18, 658-666.

45. Tricon S, Burdge GC, Jones EL, et al. (2006) Effects of dairy products naturally enriched with cis-9,trans-11 conjugated linoleic acid on the blood lipid profile in healthy middle-aged men. Am J Clin Nutr 83, 744-753.

46. Burdge GC, Tricon S, Morgan R, et al. (2005) Incorporation of cis-9 trans-11 conjugated linoleic acid and vaccenic acid (trans$1118: 1$ ) into plasma and leucocyte lipids in healthy men consuming dairy products naturally enriched in these fatty acids. $\mathrm{Br}$ J Nutr 94, 237-243.

47. Renaville B, Mullen A, Moloney F, et al. (2006) Eicosapentaenoic acid and 3,10 dithia stearic acid inhibit the desaturation of trans-vaccenic acid into cis-9 trans-11-conjugated linoleic acid through different pathways in Caco-2 and T84 cells. Br J Nutr 95, 688-695.

48. Reynolds CM, Loscher CE, Moloney AP, et al. (2008) Cis-9 trans-11-conjugated linoleic acid but not its precursor trans-vaccenic acid attenuate inflammatory markers in the human colonic epithelial cell line Caco-2. Br J Nutr 100, 13-17.

49. Lock AL, Corl BA, Barbano DM, et al. (2004) The anticarcinogenic effect of trans-11 18:1 is dependent on its conversion to cis-9 trans-11 CLA by delta9-desaturase in rats. J Nutr 134, $2698-2704$

50. Moss J (2006) Labeling of trans fatty acid content in food, regulations and limits - the FDA view. Atheroscler Suppl 7, 57-59.

51. Canadian Food Inspection Agency (2005) Information letter: Labelling of trans fatty acids. Canadian Food Inspection Agency. http://www.inspection.gc.ca/english/fssa/labeti/inform/ transe.shtm (accessed 14 February 2007). 